\title{
BUBBLEDECK: INOVAÇÃO, SUSTENTABILIDADE E CUSTO- BENEFÍCIOS NO SISTEMA CONSTRUTIVO DE LAJES BUBBLEDECK
}

\section{BUBBLEDECK: INNOVATION, SUSTAINABILITY AND COST-BENEFIT IN THE BUBBLEDECK SLAB CONSTRUCTIVE SYSTEM}

\author{
Juliana Pimentel Dialaman ${ }^{1}$ \\ Lucas Rodrigo Miranda ${ }^{2}$
}

Fabiana Florian $^{3}$

https://doi.org/10.47820/recima21.v2i10.956

\begin{abstract}
RESUMO
O trabalho tem por objetivo apresentar o modelo de laje bubbledeck de origem dinamarquesa. Ele é composto pela incorporação de esferas plásticas igualmente distribuídas entre telas de aço, formando um vácuo em seu interior, eliminando parte do volume de concreto que não exerce função estrutural, resultando em uma laje mais leve e resistente, tornando-se assim uma alternativa inovadora diante às lajes de concreto tradicionais. Há diversas vantagens em sua utilização, dentre elas a redução de custos, a minimização dos prazos e tempo de construção, maior eficiência, praticidade e durabilidade, e geração de maior segurança e conforto aos seus usuários. Além disso, diante da atual situação de escassez de recursos naturais e degradação do meio ambiente, há de considerar essencial a sustentabilidade presente nas esferas utilizadas, já que as mesmas são produzidas com plástico reciclável. Por meio de pesquisa de caráter explicativo e levantamento bibliográfico, tem por objetivo apresentar as vantagens e desvantagens da utilização do Sistema bubbledeck, levando em consideração desde o processo construtivo de sua estrutura e suas dimensões utilizadas.
\end{abstract}

Palavras-chave: bubbledeck, Laje, Sistema bubbledeck.

\footnotetext{
${ }^{1}$ Graduanda do Curso de Engenharia Civil da Universidade de Araraquara- UNIARA. Araraquara-SP. Email: julianapdialaman@gmail.com

${ }^{2}$ Orientador. Docente Curso de Engenharia Civil da Universidade de Araraquara- UNIARA. AraraquaraSP.E-mail: lmiranda@uniara.edu.br

${ }^{3}$ Orientadora. Doutora em Alimentos e Nutrição. Docente do curso de Engenharia Civil da UNIARA. Email: fflorian_@uniara.com
} 


\begin{abstract}
The work aims to present the bubbledeck slab model of Danish origin. It is composed by the incorporation of plastic spheres evenly distributed between steel screens, forming a vacuum inside, eliminating the part of the concrete volume that does not have a structural function, executed in a lighter and more resistant slab, thus becoming an alternative innovative compared to traditional concrete slabs. There are several advantages in its use, among them the reduction of costs, the minimization of construction deadlines and time, greater efficiency, practicality and durability, and the generation of greater safety and comfort for its users. Furthermore, given the current situation of scarcity of natural resources and environmental degradation, the sustainability present in the areas used must be considered essential, as they are produced with recyclable plastic. Through explanatory research and literature review, it aims to present the benefits and disadvantages of using the bubbledeck system, taking into account the constructive process of its structure and dimensions used.
\end{abstract}

Key-words: Bubbledeck, Slab, BubbleDeck System.

\title{
1. INTRODUÇÃO
}

O Sistema construtivo bubbledeck é um modelo de lajes mais leves, flexíveis e sustentáveis. De origem dinamarquesa, seu primeiro registro de implantação foi nos anos 2.000, em Rotterdam, na Holanda. Contando com projetos presentes em mais de 30 países. (SILVA, Yuri 2021).

"Este modelo de laje é composto pela incorporação de esferas plásticas igualmente distribuídas entre telas de aço, conhecidas como treliças, formando um vácuo em seu interior, eliminando assim, parte do volume de concreto que não exerce função estrutural, obtendo-se uma laje mais leve e resistente." (GUEDES, D. N e ANDRADE, L. R. de (2021)).

Estima-se que a redução de peso de uma laje normal para uma do modelo bubbledeck gira em torno de 35\%, e 25\% de redução na quantidade de concreto. Para cada $1 \mathrm{~kg}$ de plástico utilizado nas esferas, estima-se economizar $60 \mathrm{~kg}$ de concreto. Além disso, é preciso destacar a velocidade de execução da laje e simplificação dos materiais empregados, quando comparados a metodologias convencionais (BUBBLEDECK, 2021).

Através de sua eficiência, é possível apresentar inúmeras viabilidades da tecnologia bubbledeck, assim como: praticidade, durabilidade, redução de custos, prazos para implantação, e principalmente sua sustentabilidade ao 
reduzir a quantidade de concreto utilizada e utilização de esferas feitas com plástico reciclável. GUEDES, D. N e ANDRADE, L. R. de (2021).

Foi realizada uma revisão bibliográfica com foco em modelos de lajes bubbledeck, técnicas para implantação, especificações para o dimensionamento, sendo realizada uma comparação com vantagens e desvantagens de utilização deste sistema construtivo.

As tecnologias abordadas são novidades no mercado brasileiro, estando presentes em poucas obras, mas podem ganhar espaço em um curto período. Portanto, estão propostas alternativas possíveis para atender a necessidade atual da construção civil. ZANCHIN, R. e SANTOS, M. F. (2021).

\section{LAJES BUBBLEDECK}

Segundo BubbleDeck (2021), bubbledecké um sistema construtivo de laje inovador e sustentável, de origem dinamarquesa. Composto pela incorporação de esferas plásticas nas lajes de concreto, uniformemente espaçadas entre duas telas metálicas soldadas e fixadas em treliças.

"Atualmente no Brasil, já contamos com mais de $300.000 \mathrm{~m}^{2}$ construídos com a tecnologia bubbledeck, incluindo projetos premiados, como a ampliação do estacionamento do Aeroporto Tom Jobim no Rio de Janeiro. Além disso, em âmbito internacional, há mais de $5.000 .000 \mathrm{~m}^{2}$ construídos com a tecnologia bubbledeck nos mais importantes mercados ao redor do mundo.

Com a proposta de ser um método revolucionário de redução de materiais em estruturas de concreto, a tecnologia bubbledeck surge como uma nova vertente para o mercado de construção civil, proporcionando uma nova forma de construir e propiciando a abertura de novas redes de relacionamento, a fabricação de novos insumos e o desenvolvimento de novos softwares, aplicativos e equipamentos capazes de auxiliar o processo construtivo com sustentabilidade e inovação." (BubbleDeck, 2021). 


\subsection{SISTEMA CONSTRUTIVO BUBBLEDECK}

De acordo com Guedes, D. N. (2015), o conceito de lajes bubbledeck foi desenvolvido na Dinamarca, em meados da década de 1980, pelo engenheiro dinamarquês Jorgen Breuning em um concurso nacional com o objetivo de desenvolver soluções ecológicas e econômicas para construções que também fossem flexíveis e aplicáveis em larga escala.

Este sistema construtivo é formado por esferas plásticas que são combinadas com malhas de aço superiores e inferiores para formar pré-lajes inferior de bubbledecks, criando módulos pré-fabricados. Essas pré-lajes podem já incluir reforços necessários para os momentos fletores. Quando os módulos não são empregados com a pré-laje são denominados de módulos básicos. GUEDES, D. N e ANDRADE, L. R. (2021).

Os módulos pré-fabricados bubbledeck são produzidos despejando-se uma camada de concreto no módulo básico. No local final de posicionamento, os elementos individuais são então ligados através de barras de ligação simplesmente posicionada nas juntas entre os elementos. Após a colocação das armaduras complementares, a camada final de concreto é despejada e curada. (GUEDES, D. N e ANDRADE, L. R. 2021).

\subsection{TIPOS DE LAJE BUBBLEDECK}

A tecnologia BubbleDeck, desenvolvida na década de 1990 por Jorgen Bruenig na Dinamarca é um tipo de laje ocada biaxial, o sistema é composto por esferas vazias feitas de plásticos, essas esferas ocas ocupam os locais que desempenham a função estrutural da laje de menos intensidade. As esferas são colocadas uniformemente entre as armaduras de aço inferiores e superiores de forma a preencher os espaços com as bolhas ocas onde seria preenchidos por concreto porem que de nada contribuiria para a resistência final da peça, reduzindo o peso próprio da laje de 25 a 35\% se for comparado com uma laje maciça de mesmo dimensionamento. Assim contribuindo para que o projeto tenha vãos maiores, menor seção dos pilares, e menos sobrecargas nas fundações das edificações, é estimado que com a utilização deste sistema onde 
1 (um) Kg de bolhas plásticas faz com que a economia de concreto chega a ser de até $100 \mathrm{~kg}$ em uma laje de mesma espessura. (ARCHDAILY, 2021).

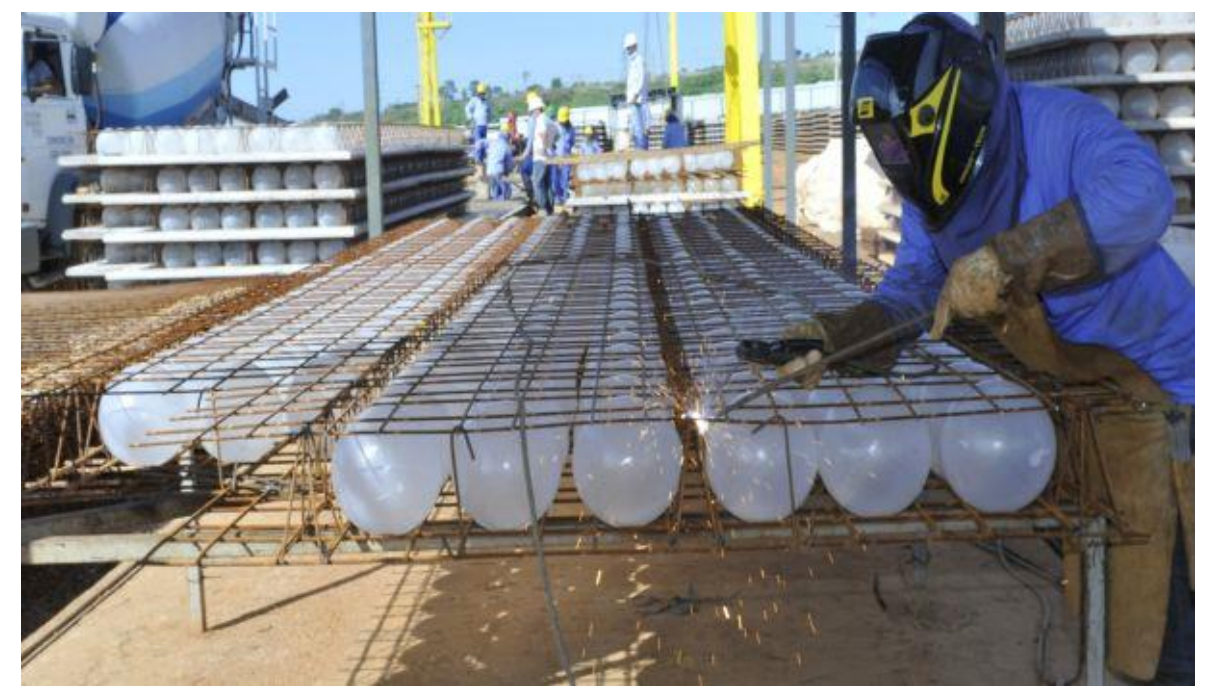

Figura 1 - Módulo bubbledeck

Fonte: (FILES, 2021)

Para formar um painel pre moldado se faz uma camada na parte inferior do modulo de $6 \mathrm{~cm}$ que pode ser utilizado como o próprio assoalho.

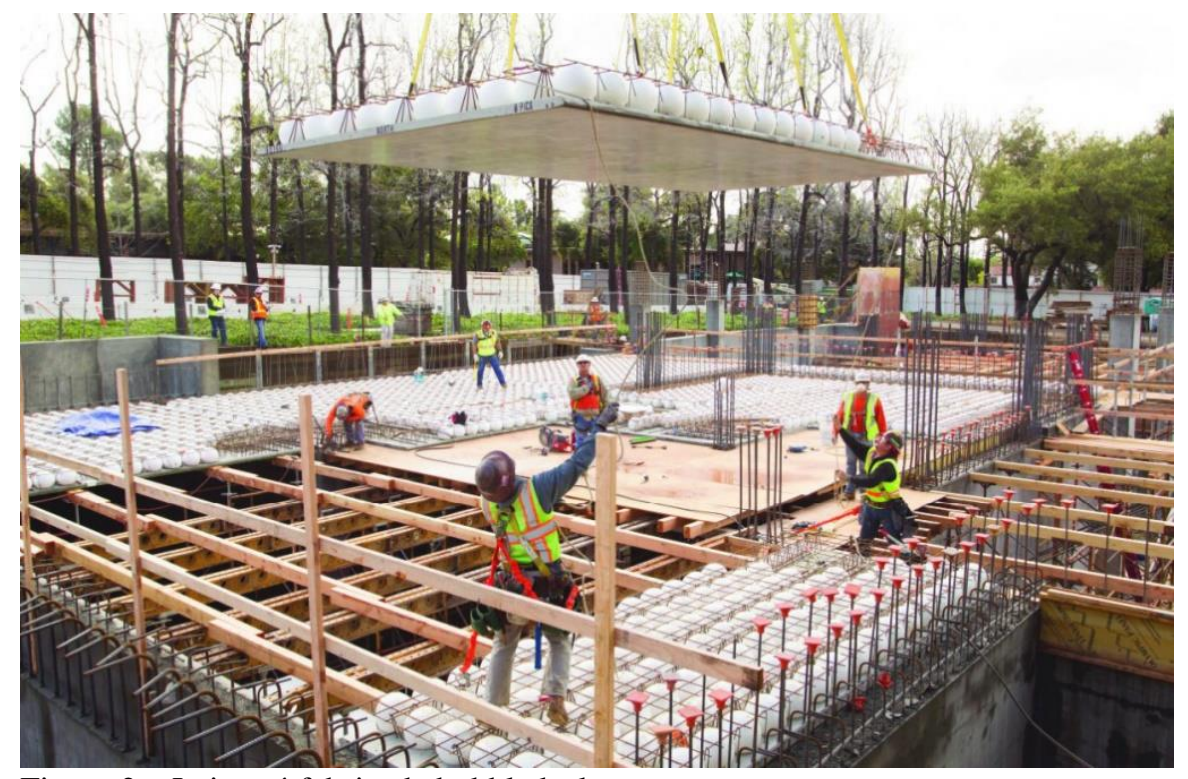

Figura 2 - Laje pré-fabricada bubbledeck

Fonte: (ARCHDAILY 2021) 
O terceiro método de construção da laje bubbledeck compreende os painéis acabados. (INOVACIVIL, 2021).

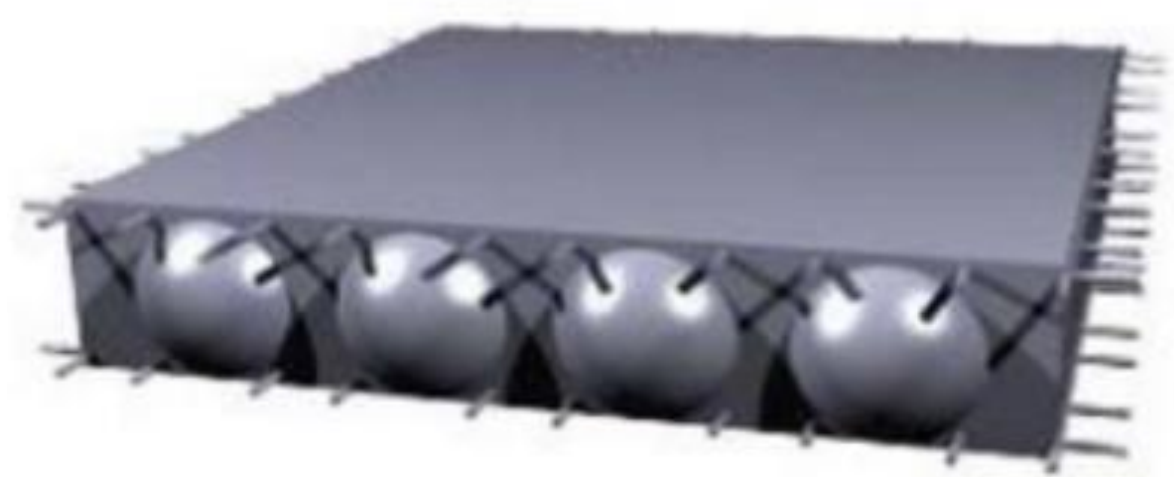

Figura 1- Painel acabado em bubbledeck

Fonte: (INOVACIVIL, 2021)

\section{TÉCNICAS PARA UTILIZAÇÃO}

Para Palla, F. 2017, os sistemas bubbledeck que envolvem a concretagem in loco, todo o detalhamento das ligações e especificações podem seguir os critérios de projeto e execução iguais das lajes maciças. A utilização conjunta de outras técnicas de construção também é possível, como por exemplo, o concreto protendido. Em suma, a sequência de execução usual dos painéis são: (DOCPLAYER, 2021).

> Uso de escoramento provisório;

$>$ Os capitéis que é uma armadura adicional superior caso necessário;

> A colocação de armadura ao longo da laje que é denominado reforço periférico caso precise;

$>$ Os painéis bubbledeck são manuseados com o auxílio de equipamentos mecânicos;

> Para a ligação entre as peças é utilizada juntas de reforços; 
A concretagem e o adensamento do concreto de segundo estágio para a remoção do escoramento;

> Não é necessário trabalho adicional para dar acabamentos as peças.

\subsection{DIMENSIONAMENTO}

De acordo com Parsianello, A. T. A. para escolha do tipo de laje bubbledeck o primeiro critério utilizado é o de limitação de flechas. Portanto, o vão máximo utilizado para cada tipo de laje é determinado pela razão entre o menor comprimento (L) do vão e a espessura (d) da laje (L/d). O fabricante sugere em seu manual as seguintes razões de L/d para os diferentes tipos de vãos: $L$ d balanço $L$ d vãos contínuos $L$ d vãos simples. (REPOSITORIO, 2021)

Também de acordo com o vão, a Figura 4. Apresenta a espessura padronizada das lajes bubbledeck associada à sua carga permanente equivalente. (REPOSITORIO, 2021).

\begin{tabular}{|l|c|c|c|c|r|}
\hline Tipo & Espessura da Laje & Diâmetro das Esferas & Vão & Carga(PP) & Concreto \\
\hline & {$[\mathbf{m m}]$} & {$[\mathbf{m m}]$} & {$[\mathbf{m}]$} & {$\left[\mathbf{k g} / \mathbf{m}^{\mathbf{2}}\right]$} & {$\left[\mathbf{m}^{\mathbf{3}} / \mathbf{m}^{\mathbf{2}}\right]$} \\
\hline BD230 & $\mathbf{2 3 0}$ & $\phi \mathbf{1 8 0}$ & $\mathbf{7 ~ a ~ 1 0}$ & 370 & 0,10 \\
\hline BD280 & $\mathbf{2 8 0}$ & $\phi \mathbf{2 2 5}$ & $\mathbf{8}$ a 12 & 460 & 0,14 \\
\hline BD340 & $\mathbf{3 4 0}$ & $\phi \mathbf{2 7 0}$ & $\mathbf{9}$ a 14 & 550 & 0,18 \\
\hline BD390 & $\mathbf{3 9 0}$ & $\phi \mathbf{3 1 5}$ & $\mathbf{1 0}$ a 16 & 640 & 0,20 \\
\hline BD450 & $\mathbf{4 5 0}$ & $\phi \mathbf{3 6 0}$ & $\mathbf{1 1}$ a 18 & 730 & 0,25 \\
\hline
\end{tabular}

Figura 2 - Vãos usuais e sua carga permanente equivalente Fonte: (DOCPLAYER, 2021)

A capacidade de redução de carga das esferas pode variar, assim como seus inter eixos, e ambos os fatores dependem da taxa de esferas por metro quadrado na laje. Abaixo na Figura 5 são apresentados os diâmetros padronizados das esferas e alguns valores relacionados a cada diâmetro. (Parsianello, A. T. A, 2021). 


\begin{tabular}{|l|c|c|c|c|c|c|c|}
\hline Diâmetro da Esfera [cm] & 18,00 & 22,50 & 27,00 & 31,50 & 36,00 & 40,50 & 45,00 \\
\hline Mínimo Intereixo das Esferas [cm] & 20,00 & 25,00 & 30,00 & 35,00 & 40,00 & 45,00 & 50,00 \\
\hline Máximo Número de Esferas [1/ $\left.{ }^{2}\right]$ & 25,00 & 16,00 & 11,00 & 8,16 & 6,25 & 4,94 & 4,00 \\
\hline Espessura Mínima da Laje [cm] & 23,00 & 28,00 & 34,00 & 40,00 & 45,00 & 52,00 & 58,00 \\
\hline Redução de Carga Por Esfera [kN] & 0,08 & 0,15 & 0,26 & 0,41 & 0,61 & 0,87 & 1,19 \\
\hline Redução Máx. de Carga $/ \mathrm{m}^{2}\left[\mathrm{kN} / \mathrm{m}^{2}\right]$ & 1,91 & 2,39 & 2,86 & 3,34 & 3,82 & 4,29 & 4,77 \\
\hline Fator para Rigidez [-] & 0,88 & 0,87 & 0,87 & 0,88 & 0,87 & 0,88 & 0,88 \\
\hline Fator para o Cortante [-] & 0,60 & 0,60 & 0,60 & 0,60 & 0,60 & 0,60 & 0,60 \\
\hline Fator para o Peso [-] & 0,67 & 0,66 & 0,66 & 0,67 & 0,66 & 0,67 & 0,67 \\
\hline
\end{tabular}

Figura 3 - Especificações Técnicas

Fonte: (DOCPLAYER, 2021)

Abaixo na figura 6 pode ser observado as especificações e dados técnicos quanto a determinação para o tipo de estrutura a ser empregado pelo projetista quanto ao dimensionamento, apresentando todas as espessuras disponíveis deste tipo de laje. (OLIVEIRA, G. H. 2021).

\begin{tabular}{cccccc}
\hline TIPO & $\begin{array}{c}\text { ESPESSURA } \\
\text { DA LAJE }(\mathbf{m m})\end{array}$ & $\begin{array}{c}\text { DIÂMETRO } \\
\text { DAS ESFERAS } \\
(\mathbf{m m})\end{array}$ & $\begin{array}{c}\text { CARGA } \\
\text { TOTAL } \\
(\mathbf{k P a})\end{array}$ & $\begin{array}{c}\text { CONSUMO DE } \\
\text { CONCRETO } \\
\left(\mathbf{m}^{\mathbf{3}} \mathbf{\mathbf { m } ^ { 2 }}\right)\end{array}$ & $\begin{array}{c}\text { VÃOS MÁXIMOS A } \\
\text { SEREM VENCIDOS } \\
(\mathbf{m})\end{array}$ \\
\hline BD230 & 230 & 180 & 4,26 & 0,15 & $6-9$ \\
\hline BD285 & 285 & 225 & 5,11 & 0,19 & $7-11$ \\
\hline BD340 & 340 & 270 & 6,22 & 0,23 & $9-13$ \\
\hline BD395 & 395 & 315 & 6,92 & 0,25 & $10-15$ \\
\hline BD450 & 450 & 360 & 7,95 & 0,31 & $11-17$ \\
\hline
\end{tabular}

Figura 4 - Comparação entre os tipos de estruturas bubbledeck Fonte: (DOCPLAYER, 2021)

\section{VANTAGENS}

Os profissionais da área da construção civil, podem se beneficiar e muito com este método, podendo ter mais liberdade nos projetos com layouts flexíveis facilmente adaptando a layouts irregulares e curvos, com a redução do peso próprio 35\% menor e podendo ter um aumento dos inter-eixos das colunas em até $50 \%$ a mais do que estruturas tradicionais, adquirindo maior rapidez e economia pela eliminação das vigas e, consequentemente pela ausência do serviços de alvenaria e instalação, com a eliminação de paredes de apoio o que facilita a metodologia construtiva (WORDPRESS, 2021).

Uma redução significativa do volume de concreto onde $1 \mathrm{~kg}$ substitui em média $60 \mathrm{~kg}$ de concreto, ambientalmente adequado na redução de energia e 
emissão de $\mathrm{CO} 2$, muitos são os aspectos positivos que vão desde atenuação do nível de ruído entre pavimentos, desempenho acústico em conformidade com Norma de Desempenho 15.575/ABNT, apresenta também condutibilidade térmica reduzida. (WORDPRESS, 2021).

Detém "Selo Verde" com prêmios internacionais, não apenas por reduzir as quantidades de materiais empregados em uma mesma área construída, mas consequentemente reduzindo a emissão de $\mathrm{CO} 2$ na atmosfera, mas também por utilizar plástico reciclável em substituição ao concreto. (WORDPRESS, 2021).

É uma Tecnologia que se enquadra nas referências preconizadas pelo Tratado de Kyoto e pelo COP15. (WORDPRESS, 2021).

\section{RESULTADOS}

Os principais resultados iniciam a partir das esferas plásticas inseridas na estrutura, que atuam como elemento de preenchimento, reduzindo o peso total da laje. O sistema possuí resistência a tração e compressão, não causa problemas estruturais e apresenta maior durabilidade. Em relação aos custos da obra, são reduzidos devido ao inferior consumo de concreto, com menores números de vigas e paredes de apoio. $O$ tempo de obra também diminui gradativamente, devido ao processo de execução na fabricação das lajes. Há redução na emissão de gases e conta com a utilização de materiais reciclados.

Através da comparação com as lajes convencionais realizada e apresentada no canal CalculistasClub (2021), há estimativas de reduções: 67\% em escoramentos, $95 \%$ em utilização de formas, $69 \%$ em quantidade total de concreto lançado in loco, $41 \%$ no valor total de armações. Neste estudo realizado, a obra trabalhou com dois turnos de 19 horas diárias, resultando na produção de $1200 \mathrm{~m}^{2}$ em 4,5 dias. Totalizou cerca de $6857 \mathrm{~m}^{2} / \mathrm{mês}$ no período de 7 meses de execução. Nesse contexto, todo o sistema bubbledeck apresentou resultados satisfatórios em todos os sentidos.

É importante ressaltar, que a tecnologia Bubbledeck ganhou diversos prêmios europeus em razão do alto grau de inovação e sustentabilidade. No Brasil, temos um fornecedor qualificado que atende aos requisitos, a empresa Bubbledeck Brasil que apresenta mão de obra qualificada para projetar os 
cálculos são baseados do mesmo modo das lajes maciças, com a utilização da norma NBR 6118:2014, executando o sistema em diversos empreendimentos.

\section{CONCLUSÃO}

Podemos concluir que o sistema construtivo bubbledeck é uma solução inovadora para construção de lajes, e possui várias vantagens ao adquiri-lo, sendo elas: menor custo, diminuição no tempo de execução, durabilidade da estrutura e sustentabilidade.

No Brasil, para estruturas pequenas, pode não ser tão viável devido ao deslocamento de uma equipe qualificada para sua implantação, mas para grandes construções o método já provou ser mais eficiente, acarretando até na elevação do número de andares que a estrutura acaba suportando.

Devido à escassez de matéria prima, diminuição de emissão de gases e utilização de materiais reciclados, o modelo de laje bubbledeck será futuramente uma das melhores soluções encontradas para fabricação de lajes em todos os continentes, surgindo novas tecnologias, normas de segurança e mão de obra mais qualificadas para atender toda a demanda mundial. 


\section{REFERÊNCIAS BIBLIOGRÁFICAS}

ARCHDAILY - Figura 2 - Laje pré-fabricada bubbledeck - Inovação + sustentabilidade. Disponível em:

https://www.archdaily.com.br/br/945876/lajes-com-bolhas-de-ar-como-osistema-bubbledeck-funciona >Acesso em 14.10.2021.

Associação brasileira de normas técnicas (ABNT), NBR 6118:2014, Projeto de Estruturas de Concreto Armado. >Acesso em 11.08.2021.

BUBBLEDECK BRASIL. Bubbledeck - Inovação + sustentabilidade. Disponível em: https://www.bubbledeck.com >Acesso em: 04.05.2021.

BUBBLEDECK BRASIL. Inovação + sustentabilidade. Disponível em: https://www.bubbledeck.com.br/quem-somos/ >Acesso em 23.05.2021.

BUBBLEDECK INTERNACIONAL. Inovação + sustentabilidade. Disponível em: BubbleDeck - Lighter Flat Slab Technology (bubbledeck-uk.com) >Acesso em: 26.04.2021.

BUBBLEDECK-UK (2007). BubbleDeck Engineering Design \& Properties Overview. BubbleDeck UK Ltd. >Acesso em: 04.05.2021.

CalculistasClub. Inovação + sustentabilidade. Disponível em:

https://www.youtube.com/watch?v=yeOq7913YWk >Acesso em 18.09.2021.

CÃLIN, Sergiu et al. Sumary of tests and studies done abroad on the bubble deck system. Department of Concrete, Technology and Organization, 2009. >Acesso em: 04.05.2021.

CEBALLOS, Manuel Alejandro. Análise Experimental à punção em lajes tipo Bubbledeck com armadura de cisalhamento. Distrito Federal, Brasília, 2017. >Acesso em: 04.07.2021.

DOCPLAYER - PALLA, F. REFERENCIAL TEÓRICO E ESTUDO COMPARATIVO DO DIMENSIONAMENTO DE LAJES BUBBLEDECK. Monografia de projeto final em engenharia civil. Universidade Federal de Goiás, GO. >Acesso em: 04.05.2021. 
DIN 1045, 2001, Concrete - German code for the design of concrete structures, 2001. >Acesso em 11.08.2021.

DIMENSIONAMENTO - Inovação + sustentabilidade. Disponível em: https://files.cercomp.ufg.br/weby/up/140/o/REFERENCIAL TE\%C3\%93RICO E ESTUDO COMPARATIVO DO DIMENSIONAMENTO DE LAJES BUBBL EDECK.pdf $>>$ Acesso em 14.10.2021.

DOCPLAYER - Figura 5 - Especificações Técnicas - Inovação + sustentabilidade. Disponível em: https://docplayer.com.br/docsimages/43/8963929/images/page_17.jpg >Acesso em 14.10.2021.

DOCPLAYER - Figura 6 - Vãos usuais - Inovação + sustentabilidade.

Disponível em: https://docplayer.com.br/docsimages/43/8963929/images/page 17.jpg >Acesso em 14.10.2021.

DOCPLAYER - Comparativo - Inovação + sustentabilidade. Disponível em: https://docplayer.com.br/84486790-Referencial-teorico-e-estudo-comparativodo-dimensionamento-de-lajes-bubbledeck.html >Acesso em 14.10.2021.

EN 13747,2005, Concrete - Precast concrete products - Floor plates for floor systems, 2005. >Acesso em 11.08.2021.

FILES - Figura 1 - Módulo bubbledeck - Inovação + sustentabilidade. Disponível em: https://civilizacaoengenheira.files.wordpress.com/2013/09/cadf2.jpg >Acesso em 14.10.2021.

GUEDES, D. N e ANDRADE, L. R. AVALIAÇÃO DE DESEMPENHO DE ESTRUTURAS UTILIZANDO LAJES DO TIPO BUBBLEDECK. MONOGRAFIA DE PROJETO FINAL EM ENGENHARIA CIVIL, Departamento de Engenharia Civil e Ambiental, Universidade de Brasília, Brasília, DF. >Acesso em: 11.09.2021.

INOVACIVIL - Figura 7- Painel acabado - Inovação + sustentabilidade. Disponível em:

https://www.inovacivil.com.br/lajes-bubbledeck-entenda-como-economizarconcreto-com-bolhas/ >Acesso em 14.10.2021.

LAI, Tina. Structural Behavior of BubbleDeck Slabs And Their Application to Lightweight Bridge Decks. Department of Civil and Environmental Engineering. Massachusetts Institute of Technology. June 2010. >Acesso em: 04.05.2021. 
LIMA, H. J. N. Análise experimental à punção de lajes lisas tipo BubbleDeck. Dissertação de Mestrado em Estruturas e Construção Civil, Publicação E.DM 002 A/15, Departamento de Engenharia Civil e Ambiental, Universidade de Brasília, Brasília, DF, 113p. >Acesso em: 04.05.2021.

NICÁCIO, Wanderley Gustavo. Comportamento à punção de lajes de concreto armado tipo bubbledeck, Brasília, 2018. >Acesso em: 04.05.2021.

NORMAS TÉCNICAS - Associação brasileira de normas técnicas (ABNT), NBR 6118, 2003, Projeto e Execução de Obras de Concreto Armado >Acesso em 11.08.2021.

OLIVEIRA, G. H. LAJES BUBBLEDECK - Inovação + sustentabilidade. Disponível em:

https://www.researchgate.net/publication/346985796 Lajes bubbledeck um C omparativo com o sistema construtivo de lajes macicas. >Acesso em 11.08.2021.

PARSIANELLO, A. T. PLANEJAMENTO E EXECUÇÃO DE LAJES BUBBLEDECK NA OBRA: Estudo de Caso. Trabalho de Curso (TC), UniCEUB - Centro Universitário de Brasília. >Acesso em: 04.05.2021.

REPOSITORIO - Critérios - Inovação + sustentabilidade. Disponível em: https://repositorio.uniceub.br/jspui/bitstream/235/6411/1/21032146.pdf $>$ Acesso em 14.10.2021.

REPOSITORIO - Critérios - Inovação + sustentabilidade. Disponível em: https://pantheon.ufrj.br/bitstream/11422/9646/1/monopoli10006941.pdf $>$ Acesso em 14.10.2021.

SILVA, Yuri de Oliveira. Estudo comparativo entre lajes "BubbleDeck" e lajes lisas. Projeto de graduação. Rio de Janeiro: Universidade Federal do Rio de Janeiro - UFRJ, 2011. >Acesso em: 04.05.2021.

SOUZA, F. S. T. BUBBLEDECK - Inovação + sustentabilidade. Disponível em: https://www.nossocanteirodeobra.com.br/bubbledeck-o-futuro-da-construcaocivil/ >Acesso em 27.05.2021. 
WORDPRESS - As Vantagens - Inovação + sustentabilidade. Disponível em: https://civilizacaoengenheira.wordpress.com/2017/03/24/bubbledeck-asesferas-plasticas-como-sustentabilidade-para-a-construcao-civil/ $>$ Acesso em 14.10.2021.

ZANCHIN, R. e SANTOS, M. F. - Inovação + sustentabilidade. Disponível em: https://revistaseletronicas.fmu.br/index.php/inovae/article/view/2030 >Acesso em 11.08.2021.

https://pt.slideshare.net/GuilhermeCamargos/as-esferas-plsticasbubbledeck-para-construo-civil >Acesso em 11.08.2021.

https://www.confea.org.br/sites/default/files/uploads-

imce/Contecc2019/Civil/ANALISE\%20DO\%20DESEMPENHO\%20DA\%20LAJE \%20BUBBLEDECK\%20EM\%20COMPARA\%c3\%87\%c3\%830\%20A\%20LAJE \%20NERVURADA.pdf $>$ Acesso em 11.08.2021. 